\title{
Displacement Prediction of Tunnel Surrounding Rock: A Comparison of Support Vector Machine and Artificial Neural Network
}

\author{
Qingdong Wu, ${ }^{1}$ Bo Yan, ${ }^{2}$ Chao Zhang, ${ }^{2}$ Lu Wang, ${ }^{3}$ Guobao Ning, ${ }^{4}$ and B. Yu ${ }^{5}$ \\ ${ }^{1}$ Shandong Luqiao Group CO., Ltd, Jinan 250021, China \\ ${ }^{2}$ Transportation Management College, Dalian Maritime University, Dalian 116026, China \\ ${ }^{3}$ China Academy of Civil Aviation Science and Technology, Beijing 100028, China \\ ${ }^{4}$ School of Automotive Studies, Tongji University, Shanghai 201804, China \\ ${ }^{5}$ School of Traffic and Transportation, Beijing Jiaotong University, Beijing 100044, China \\ Correspondence should be addressed to Guobao Ning; guobao_tj@163.com and B. Yu; yubjjt@126.com
}

Received 5 May 2014; Revised 13 July 2014; Accepted 14 July 2014; Published 23 July 2014

Academic Editor: Rui Mu

Copyright (C) 2014 Qingdong Wu et al. This is an open access article distributed under the Creative Commons Attribution License, which permits unrestricted use, distribution, and reproduction in any medium, provided the original work is properly cited.

\begin{abstract}
Displacement prediction of tunnel surrounding rock plays an important role in safety monitoring and quality control tunnel construction. In this paper, two methodologies, support vector machines (SVM) and artificial neural network (ANN), are introduced to predict tunnel surrounding rock displacement. Then the two modes are texted with the data of Fangtianchong tunnel, respectively. The comparative results show that solutions gained by SVM seem to be more robust with a smaller standard error compared to ANN. Generally, the comparison between artificial neural network (ANN) and SVM shows that SVM has a higher accuracy prediction than ANN. Results also show that SVM seems to be a powerful tool for tunnel surrounding rock displacement prediction.
\end{abstract}

\section{Introduction}

Limited by geographic condition, digging method, and many other factors, the loading features of tunnel construction are extremely complicated. As a result, the displacement of tunnel surrounding rock has random variations. Although the conditions of tunnel surrounding field monitoring could be gained by field monitoring, the field monitor required many resources that could not be afforded by constructors $[1,2]$. Meanwhile, tunnel surrounding rock distortion and tunnel lining crack could also lead to the instability of the tunnel during tunnel construction [3]. So it is necessary to estimate the potential tunneling problems as early as possible.

Along with the development of information technologies, a number of intelligent algorithms, such as regression analysis, gray theory, data smoothing processing, time series analysis, and genetic algorithm [4-13], have been applied to predict the displacement of tunnel surrounding rocks [14, 15]. Till now there are many literatures that have studied the displacement prediction of tunnel surrounding rock [16]. Parlos et al. [17] presented a multistep-ahead prediction for the systems of high complexity and a new method that is proved that it could be used to increase the accuracy of prediction relying on a dynamic recurrent neural network. Besides the dynamic model, Cheng et al. [18] also presented a multistep-ahead prediction model, which is not relying on a dynamic recurrent neural network but uses a method based on support vector machine (SVM) to predict and diagnose the type of long-term changes of hydropower structures. In the model, to diagnose the long-term changes of hydropower, a dynamic spline interpolation is established based on a multilayer adaptive time delayed network to ensure the prediction accuracy. In order to solve the nonlinear time series problems, Lee et al. [19] also proposed multistep-ahead model based on article neutral network to improve the robust and accuracy of predicting in solving this type of problems. And, recently, Chevillon et al. [20] proposed that not only the multistep-ahead model, but also the nonparametric direct 
multistep-ahead model could be used to predict the reliability of surrounding rock and other geographic structures. There are also some other studies that rely on multistep-ahead model to do the predicting [21, 22]. In general, in recent years, there are more and more researchers and institutions, which tend to use the multistep-ahead model to predict the complex internal structures.

Nevertheless, as many researchers stated, the accuracy of the predicting models based on the multistep-ahead model extremely relied on the similarity between the present and previous data. And considering the complexity of internal structure it is almost impossible to gain a large amount of accurate historical data. Therefore, with the unreliable historical data, the predicting results could also become seriously incorrect. Along with the development of intelligent rock mechanics, artificial neural network (ANN) technique is gradually matured and widely used. In recent years, ANN has been successfully applied in the field of tunnel surrounding rock displacement prediction. Meanwhile, thanks to the development of statistical learning theory, support vector machine (SVM), as a new machine learning technology, has drawn much attention in the research area of time series forecasting. Therefore, in the paper, two models, displacement prediction of tunnel surrounding rock based on ANN and displacement prediction of tunnel surrounding rock based on SVM, are presented. And then in order to compare the two models, in the last section the two models are evaluated with data of Fangtianchong tunnel, respectively. If the simple models presented in the paper could be applied to practice in the future, the building security and effectiveness could be definitely improved.

The objectives of this study are to examine and compare the feasibility of applying ANN and SVM to predict tunnel surrounding rock displacement. Thus, this paper is structured as follows: Section 2 provides a brief introduction on ANN and then practices ANN to predict the displacement of tunnel surrounding rock; Section 3 provides a simple introduction of SVM and then applies SVM to predict the displacement of tunnel surrounding rock; at the end of the paper, the conclusions are presented.

\section{Artificial Neural Network (ANN) for Tunnel Surrounding Rock Displacement}

Artificial neural network (ANN) is considered as one of the most effective tools that could be used to predict nonlinear problems, especially the problem with a real world background [23]. The framework of artificial neural network (ANN) requires it to gain new data constantly; the characteristic of ANN could be used to track even a little variation repeatedly. Besides the advantage, ANN also has the ability to learn from random and noisy data, which the traditional statistical techniques cannot handle very well; the ability could make them solve problems that are often resolved by extremely complex mythologies previously.

While the BP neural network [24] is an ongoing and popular academic area of ANN, which has attracted much attention of the researchers all over the world $[1,25]$, ANN

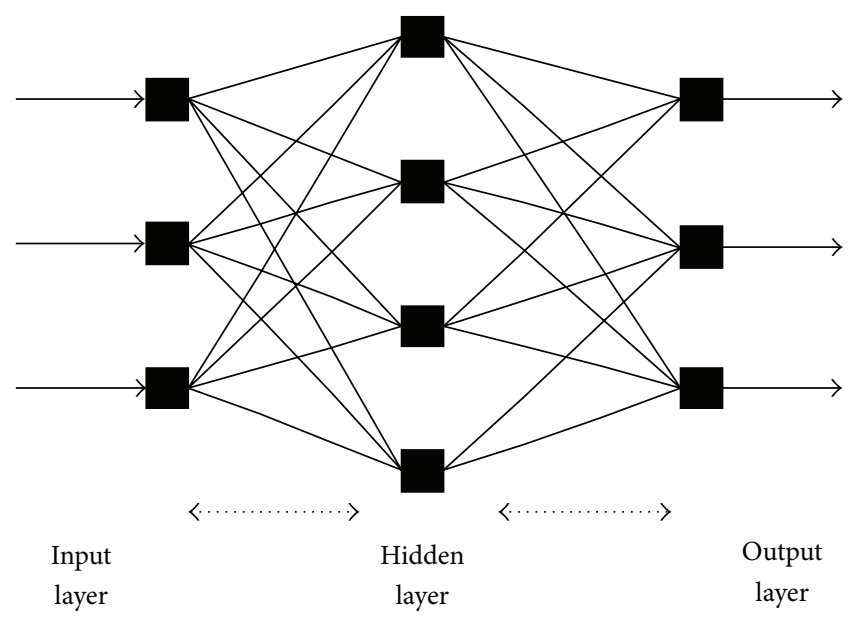

FIGURE 1: The structure of 3-layer BP neural network.

is a hierarchical feed-forward network, which also consisted of several different layers. During the training BPN would be offered by a series of input or output pattern sets over and over again. Then the network would gradually "learn" the input and output relationship by correcting the weights to mineralize the error between the actual and predicted output patterns of the training set. And the trained network would be inspected through a separate set of data, which is the test set that is used to monitor performance and validity of the model. If there is a minimum in the mean squared error (MSE), network training is completed. As shown in Figure 1, most of the BP neural networks consisted of three layers: input layer, hidden layer, and output layer.

The learning process of BP neural network for predicting tunnel surrounding rock displacement could be described as follows.

(a) Initialize the $\mathrm{BP}$ neural network by generating a random number within $[-1,1]$.

(b) Input a set of learning samples into the input layer to gain the output set of each unit of hidden layer by the following functions:

$$
\begin{aligned}
& A_{j}=\sum_{i=1}^{m} w_{i j} X_{i}-\theta_{j}, \\
& B_{j}=f\left(A_{j}\right), \quad j=1,2, \ldots, n,
\end{aligned}
$$

where $m$ is the number of the units belonging to the input layer, $n$ is the number of the units belonging to the hidden layer, $i$ could be any unit of the input layer, $j$ could be any unit of the hidden layer, $w_{i j}$ is the network link weight from any unit of the input layer to that of the hidden one, $\theta_{j}$ is the threshold of the $j$ unit of the hidden layer, and $B_{j}$ is the output value of the $j$ unit of the hidden layer.

(c) Calculate the output values of the units of the output layer. 
The output values could be calculated by the following functions:

$$
\begin{aligned}
& C_{k}=\sum_{j=1}^{n} V_{j k} B_{j}-a_{k}, \\
& D_{k}=f\left(C_{k}\right),
\end{aligned}
$$

where $l$ is the number of the units belonging to the output layer; $k$ could be any unit of the output layer; $V_{j k}$ is the network link weight from any unit of the hidden layer to that of the output one; $a_{k}$ is the threshold of the $k$ unit of the hidden layer; and $D_{k}$ is the output value of the $k$ unit of the output layer.

(d) Calculate the mean squared error (MSE) of each unit of the output layer.

$\operatorname{MSE}\left(E_{k}\right)$ could be calculated by the following functions:

$$
E_{k}=D_{k}-Y_{k},
$$

where $Y_{k}$ is the output of the training samples.

(e) Calculate the MSE of each unit of the hidden layer $\left(F_{j}\right)$ as the following functions:

$$
F_{j}=\frac{d f\left(A_{j}\right)}{d x} \sum_{k=1}^{l} V_{j k} E_{k} .
$$

(f) Based on the MSE of each unit of the output layer $E_{k}$ to adjust $V_{j k}$, that is, the network link weight from any unit of the hidden layer to that of the output one, and $a_{k}$, that is, the threshold of the $k$ unit of the hidden layer, the adjusting functions are shown as follows:

$$
\begin{aligned}
V_{j k}^{\prime} & =V_{j k}+\beta E_{k} D_{k}, \\
a_{k}^{\prime} & =a_{k}+\beta E_{k},
\end{aligned}
$$

where $\beta$ is the learning speed.

(g) Adjust $w_{i j}$, that is, the network link weight from any unit of the input layer to that of the hidden one; and adjust $\theta_{j}$, that is, the threshold of the $j$ unit of the hidden layer:

$$
\begin{gathered}
w_{i j}^{\prime}=w_{i j}+\beta F_{j} B_{j}, \\
\theta_{j}^{\prime}=\theta_{j}+\beta F_{j} ;
\end{gathered}
$$

when $E_{k}$ is less than the maximum permissible errors, the learning process is complete; if not, go back to Step (a).

\section{Support Vector Machines (SVM) for Tunnel Surrounding Rock Displacement}

SVM is a type of learning technique that is based on the structural risk minimization principle and whose objective is to minimize an upper bound of generalization error. Based on the text result from several high dimensional linear functions, SVM is shown to have very high generalization ability. Relying on the ability SVM could easily attain relatively more reliable data patterns than the traditional predicting methods.

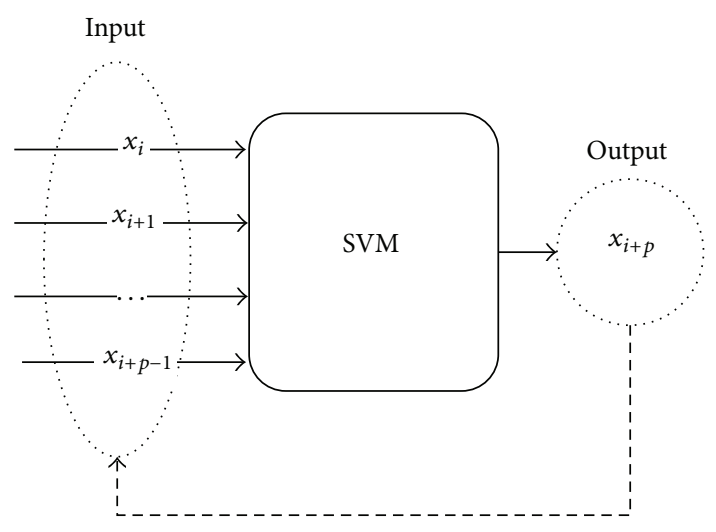

FIGURE 2: The framework of SVM.

The basic of support vector machine is shown in Figure 2 [2628].

The time sequence of tunnel surrounding rock displacement is gained by monitoring. Given time sequence data set $\left\{x_{i}\right\}=\left\{x_{1}, x_{2}, \ldots, x_{N}\right\} i=1,2, \ldots, N$, in order to predict the time sequence the relationship between $\left\{x_{i}, x_{i+1}, \ldots, x_{i+p-1}\right\}$ and the displacement value $x_{i+p}$ of the moment $i+p$ should be analyzed. The relationship is shown by the following function:

$$
x_{i+p}=f\left(x_{i}, x_{i+1}, \ldots, x_{i+p-1}\right) .
$$

According to the theory of SVM, the nonlinear relationship could be gained by learning the displacement sequence: $x_{i}, x_{i+1}, \ldots, x_{i+p-1}, i=1,2, \ldots, n-p$. SVM estimates the function by the following function:

$$
f\left(x_{n+m}\right)=\sum_{i=1}^{n-p}\left(\alpha_{i}-\alpha_{i}^{*}\right) K\left(X_{n+m}, X_{i}\right)+b,
$$

where $f\left(x_{n+m}\right)$ is the displacement value of the time $n+m, X_{n+m}=\left(x_{n+m-p}, x_{n+m-p+1}, \ldots, x_{n+m-1}\right), X_{i}=$ $\left(x_{i}, x_{i+1}, \ldots, x_{i+p-1}\right)$, and the value of $\alpha, \alpha^{*}$, and $b$ could be gained by the following function:

$$
\begin{aligned}
& \operatorname{Max} \begin{aligned}
w\left(\alpha_{i}, \alpha_{i}^{*}\right)= & -\frac{1}{2} \sum_{i, j=1}^{n-p}\left(\alpha_{l}-\alpha_{l}^{*}\right)\left(\alpha_{j}-\alpha_{j}^{*}\right) K\left(X_{i} X_{j}\right) \\
& +\sum_{i=1}^{k} x_{i+p}\left(\alpha_{l}-\alpha_{l}^{*}\right)-\varepsilon \sum_{i=1}^{n-p}\left(\alpha_{i}+\alpha_{i}^{*}\right)
\end{aligned} \\
& \text { s.t. }\left\{\begin{array}{c}
\sum_{i=1}^{n-p}\left(\alpha_{i}-\alpha_{i}^{*}\right)=0 \\
0 \leq \alpha_{i}, \quad \alpha_{i}^{*} \leq C,(i=1,2, \ldots, n-p) .
\end{array}\right.
\end{aligned}
$$

An overall description of the steps of the algorithm based on SVM is shown as follows.

(a) Initialize the BP neural network by generating a random number within $[-1,1]$.

(b) Resort the data in an ascending sort. And inspect if there are any infeasible individuals in the set; if so delete the infeasible ones and then move to next step. 
(c) In this set, $n-p$ sets should be gained from $n$ sets. Select $n$ sets from the general pool and choose $n$ of them randomly.

(d) Use competitive complex evolution algorithm, which could be called CCE algorithm, to expand the sets.

(e) A single population, which consisted of the sets provided by the former step (c), should be generated in this step.

(f) If all the conjunction standards are satisfied, then calculating process could be stopped; if not, please move to Step (g).

(g) If the number of the sets is less than $n-p$, the minimum set should be removed and then move back to Step (b).

\section{Case Study}

To further analyze and compare the predicting model for tunnel surrounding rock based on SVM and BP neural network, this paper uses the Fangtianchong tunnel of WuhanGuangzhou railway as the test bed. DK1658+905 is chosen as the typical section to attain the data of tunnel surrounding rock displacement. And in the case study the paper selects 3 sections, the first section, the second section, and the third section, as the test bed. The measurement frequency should be mentioned; the frequency of this study is decided to be once per day. The data used in this case is collected during the period from October 8 to 14, 2013. The reason why we choose this period to detect the displacement of tunnel surrounding rock is that the rock of Fantianchong is a kind of soft rock, which would be affected by the environment temperature and humidity. And from October 8 to 14 the temperature and humidity around the tunnel are stable and appropriate for detecting based on researching and constructing experiences.

In order to test the two models proposed in the paper, the total data gained by observing is divided into 3 groups, which are the testing samples, training samples, and inspection samples. In the models the 3 groups are used as three sets. The data of training and testing sets are provided by the first and third sections and the data of the inspection set is from the second section of the test bed. In this case, we use rootmean-square error (RMSE) to evaluate the predicting models mentioned in this paper. And RMSE of each day's predicting results could be gained by the following functions:

$$
\mathrm{RMSE}=\sqrt{\left\{\frac{\sum_{i-1}^{n}\left(x_{i}-\widehat{x}_{i}\right)^{2}}{n}\right\}},
$$

where the predicted value of tunnel surrounding rock displacement is $x_{i}, \widehat{x}_{i}$ is the actual value of variable tunnel surrounding rock displacement, and $n$ is the number of verifying points.

This paper compares the performance of the two models on the same test bed. The results are shown in Figure 3.

The predicting errors of SVM and ANN are shown in Figure 3; it is obvious that the errors of SVM models are much smaller than that of the model based on ANN. The comparative results show that solutions attained by SVM are more robust with a smaller standard error compared to ANN.

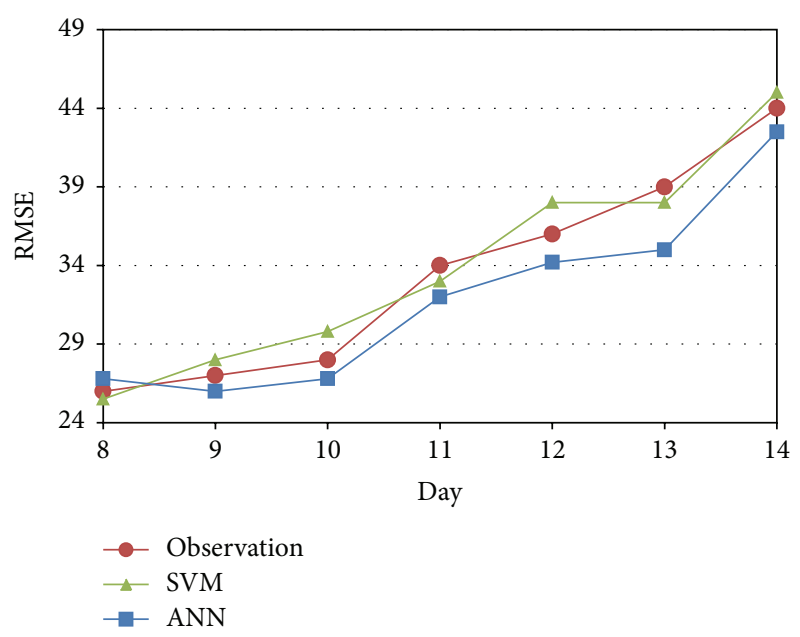

FIGURE 3: Comparison between the prediction results among observations, SVM, and ANN.

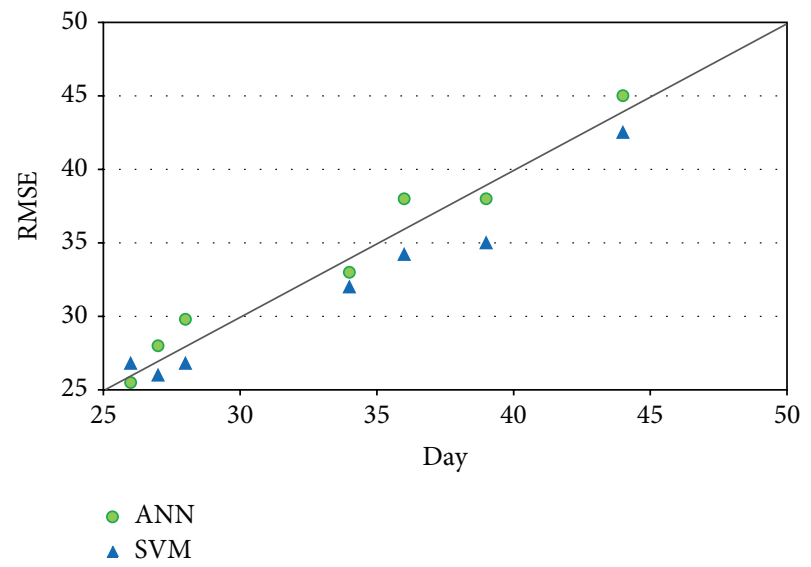

FIGURE 4: Comparison between the predicting errors of SVM and ANN.

As shown in Figure 4, with time the variations between the predicting results of ANN and the actual displacement value are small. However, the variations between the predicting results of SVM and the actual displacement value keep to be random and not in order. So it could be concluded that the structural risk minimization principle to minimize the generalization error is used by SVM, and the empirical risk minimization principle to minimize the training error is used by BP neural network. Besides, the method based on SVM tends to search for a global solution while that of BP neural network tends to fall in a certain local optimal solution. However, the predicting speed of ANN is much quicker than that of SVM. Based on our tests, the running time of the method based on SVM is about twice as much as the running time of ANN. The difference is mainly caused by the framework of SVM, which is a little more complex than the structure of ANN. Thus in the real world it is more acceptable to adapt to SVM techniques to predict the future displacements if you prefer a more accurate prediction, but 
if it is emergency it could be better to rely on ANN, whose speed is quicker and errors are acceptable.

\section{Conclusions}

Considering the potential danger that could be caused by tunnel surrounding rock displacement, it is important and necessary to have a relatively accurate and in time prediction of the displacement. Therefore, in this paper, two methodologies, support vector machines (SVM) and artificial neural network (ANN), are introduced to predict tunnel surrounding rock displacement. To compare the two models the data of Fangtianchong tunnel are applied to test the two, respectively. In general, the comparison between artificial neural network (ANN) and SVM shows that SVM has higher accuracy prediction than ANN. But the predicting speed of ANN is much quicker than that of SVM. Thus, SVM can be a potential and accurate tool for tunnel surrounding rock displacement prediction, but if it is emergency it could be better to rely on ANN, whose speed is quicker and errors are acceptable.

\section{Conflict of Interests}

The authors declare that there is no conflict of interests regarding the publication of this paper.

\section{References}

[1] C. L. Zhang, X. W. Wang, and H. Chen, "Study on forecast and prediction methods for tunnel wall rock deformation," Technology of Highway and Transport, vol. 4, pp. 88-92, 2008.

[2] W. Xiao and W. Leng, "Dynamic displacement prediction for the retaining structure of the deep excavation pit in subway," China Railway Science, vol. 25, no. 5, pp. 84-88, 2004.

[3] Q. N. Chen, Y. X. Zhang, and J. G. Chen, “The displacement prediction of surrounding rock based on BP neural network for the tunnel," Journal of Highway and Transportation Research and Development, vol. 21, no. 2, pp. 65-69, 2004.

[4] B. Z. Yao, P. Hu, X. H. Lu, J. J. Gao, and M. H. Zhang, "Transit network design based on travel time reliability," Transportation Research C: Emerging Technologies, vol. 43, part 3, pp. 233-248, 2014.

[5] B. Z. Yao, P. Hu, M. H. Zhang, and X. M. Tian, "Improved ant colony optimization for seafood product delivery routing problem," PROMET-TRAFFIC \& Transportation, vol. 26, no. 1, pp. 1-10, 2014.

[6] B. Yao, P. Hu, M. Zhang, and S. Wang, "Artificial bee colony algorithm with scanning strategy for the periodic vehicle routing problem," Simulation, vol. 89, no. 6, pp. 762-770, 2013.

[7] B. Z. Yao, J. B. Yao, M. H. Zhang, and L. Yu, "Improved support vector machine regression in multi-step-ahead prediction for rock displacement surrounding a tunnel," Scientia Iranica. In Press, http://www.scientiairanica.com/en/ArticlesInPress.

[8] B. Z. Yao, C. Y. Yang, J. B. Yao, and J. Sun, "Tunnel surrounding rock displacement prediction using support vector machine," International Journal of Computational Intelligence Systems, vol. 3, no. 6, pp. 843-852, 2010.
[9] B. Z. Yao, C. Y. Yang, J. B. Yao, J. J. Hu, and J. Sun, “An improved ant colony optimization for flexible job shop scheduling problems," Advanced Science Letters, vol. 4, no. 6-7, pp. 2127-2131, 2011.

[10] B. Yu, Z. Yang, and K. Chen, "Hybrid model for prediction of bus arrival times at next station," Journal of Advanced Transportation, vol. 44, no. 3, pp. 193-204, 2010.

[11] B. Yu, Z. Z. Yang, and B. Z. Yao, "A hybrid algorithm for vehicle routing problem with time windows," Expert Systems with Applications, vol. 38, no. 1, pp. 435-441, 2011.

[12] B. Yu, Z. Yang, and J. Yao, "Genetic algorithm for bus frequency optimization," Journal of Transportation Engineering, vol. 136, no. 6, pp. 576-583, 2010.

[13] B. Yu, H. B. Zhu, W. J. Cai, N. Ma, and B. Z. Yao, "Twophase optimization approach to transit hub location-the case of Dalian," Journal of Transport Geography, vol. 33, pp. 62-71, 2013.

[14] X. H. Li, Y. Zhao, X. G. Jin, Y. Y. Lu, and X. F. Wang, "Application of grey majorized model in tunnel surrounding rock displacement forecasting," in Advances in Natural Computation, vol. 3611 of Lecture Notes in Computer Science, pp. 584-591, 2005.

[15] Y. S. Li, X. P. Li, and C. L. Zhang, “The displacement prediction of surrounding rock based on BP neural network for the tunnel," Journal of Highway and Transportation Research and Development, vol. 25, supplement 1, pp. 2970-2973, 2006.

[16] P. J. Sellner and W. Schubert, "Prediction of displacements in tunnelling," in Proceedings of the ISRM International Symposium, International Society for Rock Mechanics, 2000.

[17] A. G. Parlos, O. T. Rais, and A. F. Atiya, "Multi-step-ahead prediction using dynamic recurrent neural networks," Neural Networks, vol. 13, no. 7, pp. 765-786, 2000.

[18] C. H. Cheng, J. Lehmann, and M. H. Engelhard, "Natural oxidation of black carbon in soils: Changes in molecular form and surface charge along a climosequence," Geochimica et Cosmochimica Acta, vol. 72, no. 6, pp. 1598-1610, 2008.

[19] H. S. Lee, H. J. Billings, and M. N. Lehman, “The Suprachiasmatic Nucleus: A Clock of Multiple Components," Journal of Biological Rhythms, vol. 18, no. 6, pp. 435-449, 2003.

[20] G. Chevillon and D. F. Hendry, "Non-parametric direct multistep estimation for forecasting economic processes, International Journal of Forecasting, vol. 21, no. 2, pp. 201-218, 2005.

[21] J. Liu, W. Wang, and F. Golnaraghi, "A multi-step predictor with a variable input pattern for system state forecasting," Mechanical Systems and Signal Processing, vol. 23, no. 5, pp. 1586-1599, 2009.

[22] B. Yu, Z. Z. Yang, and B. Yu, "Hybrid model for prediction of bus arrival times," Neural Network World, vol. 19, no. 3, pp. 321-332, 2009.

[23] B. Yegnanarayana, Artificial Neural Networks, PHI Learning, 2009.

[24] W. Jin, Z. J. Li, L. S. Wei, and H. Zhen, "The improvements of BP neural network learning algorithm," in Proceedings of the 5th International Conference on Signal Processing (WCCC-ICSP '00), vol. 3, pp. 1647-1649, IEEE, 2000.

[25] Y. Bin, Y. Zhongzhen, and Y. Baozhen, "Bus arrival time prediction using support vector machines," Journal of Intelligent Transportation Systems, vol. 10, no. 4, pp. 151-158, 2006.

[26] V. Cherkassky and Y. Ma, "Practical selection of SVM parameters and noise estimation for SVM regression," Neural Networks, vol. 17, no. 1, pp. 113-126, 2004. 
[27] C. W. Hsu, C. C. Chang, and C. J. Lin, "A practical guide to support vector classification," Tech. Rep., Department of Computer Science and Information Engineering, National Taiwan University, 2003.

[28] C. W. Hsu, C. C. Chang, and C. J. Lin, A Practical Guide to Support Vector Classification, 2003. 


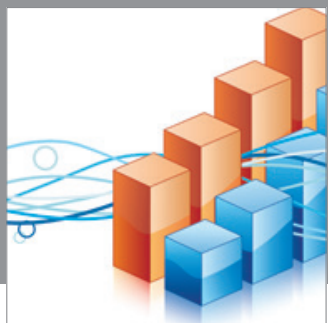

Advances in

Operations Research

mansans

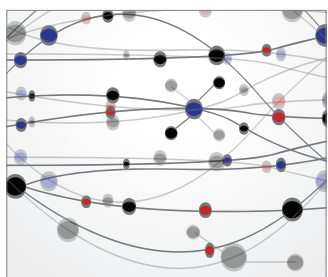

The Scientific World Journal
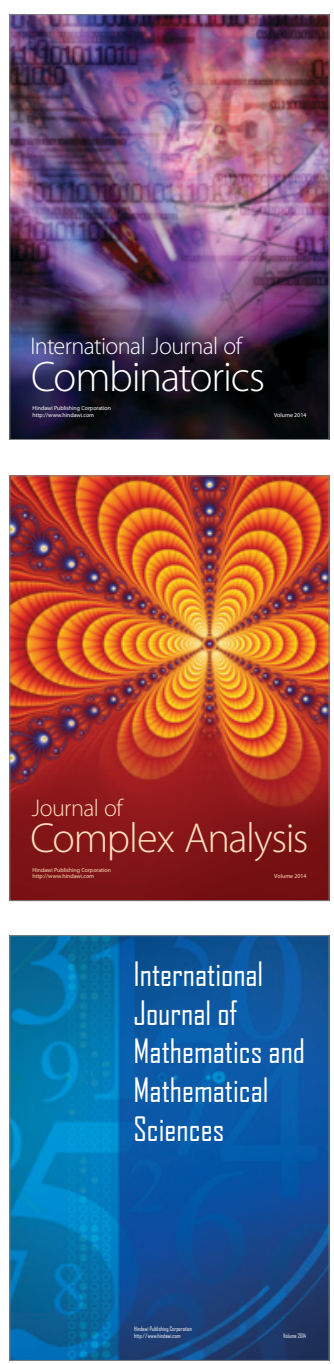
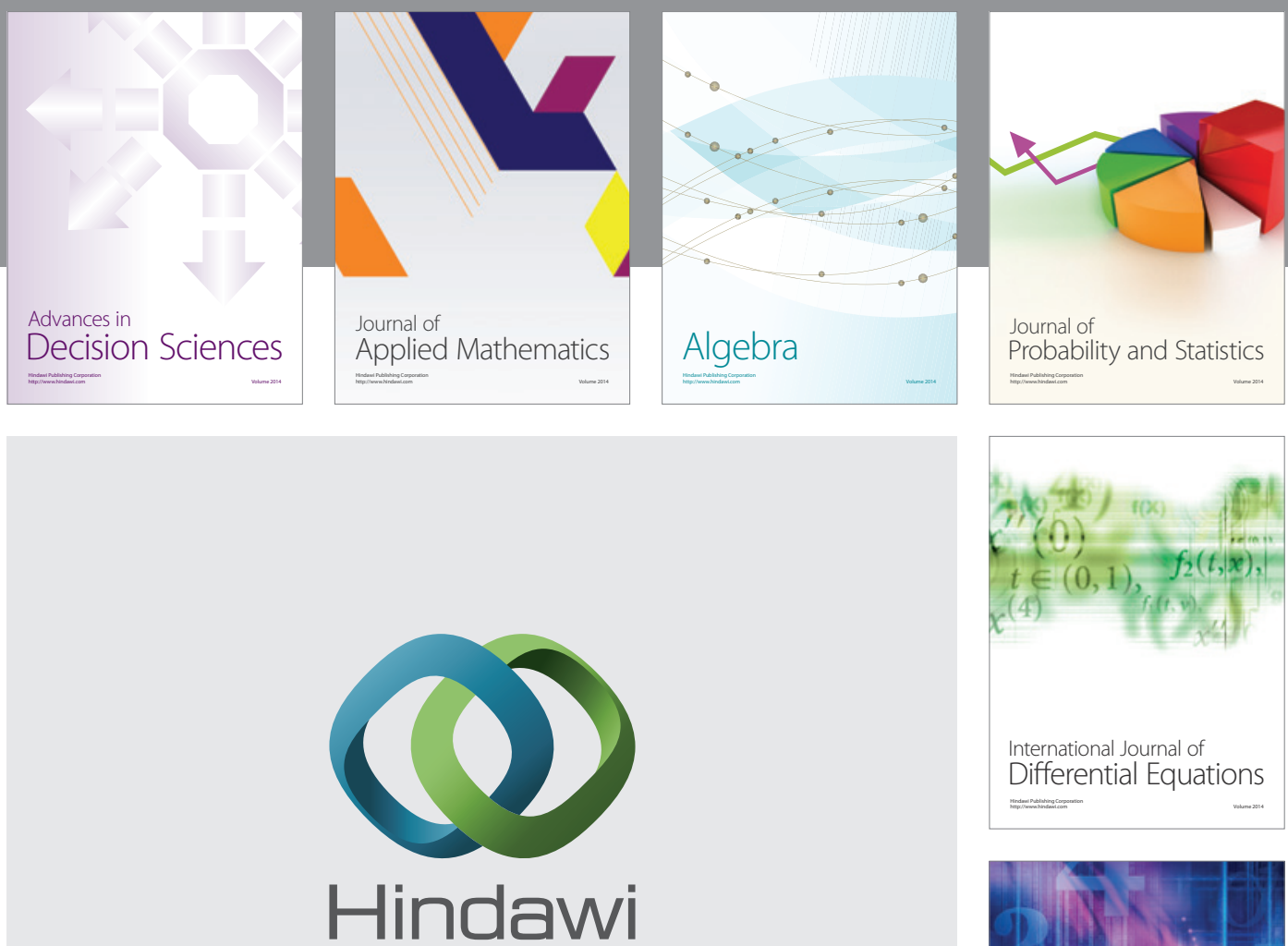

Submit your manuscripts at http://www.hindawi.com
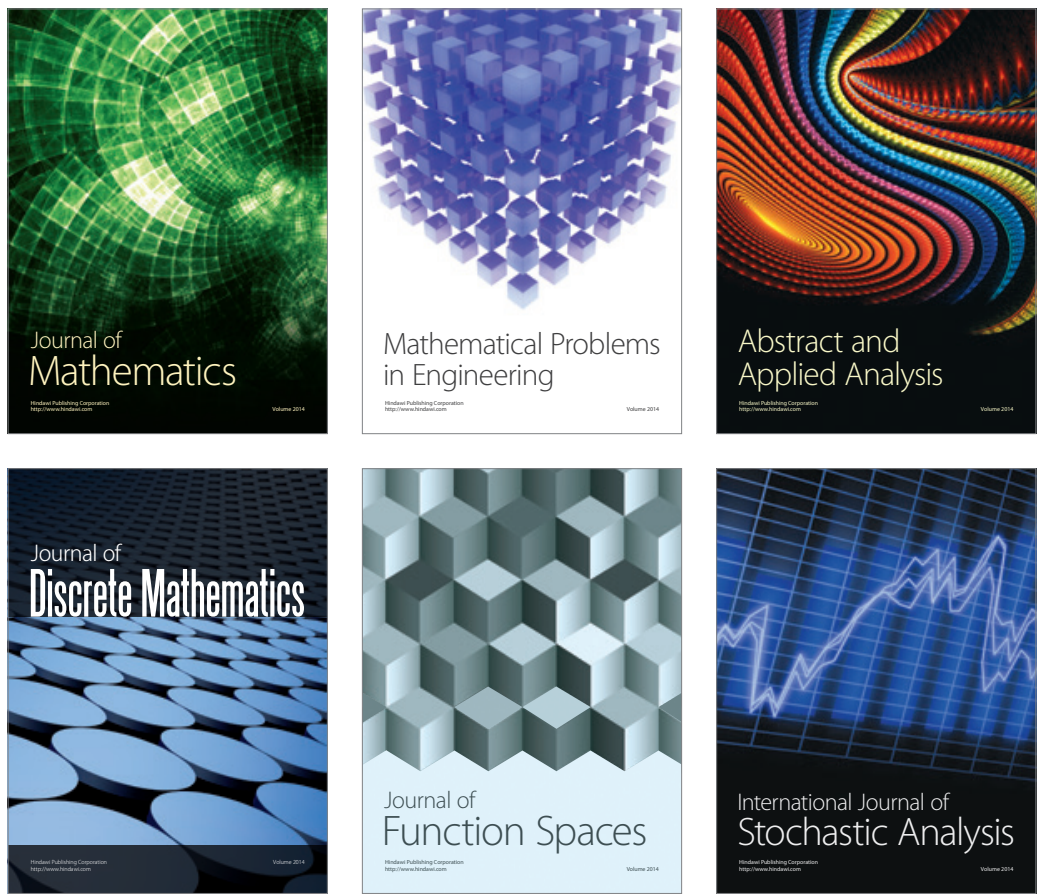

Journal of

Function Spaces

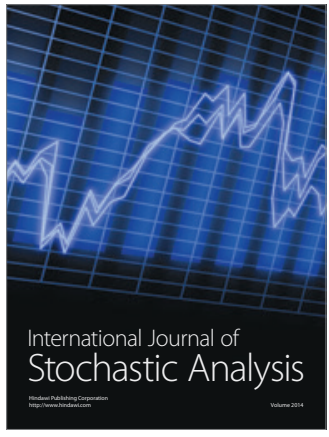

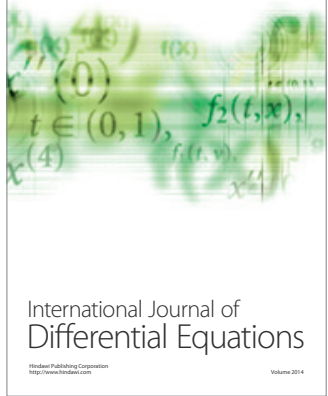
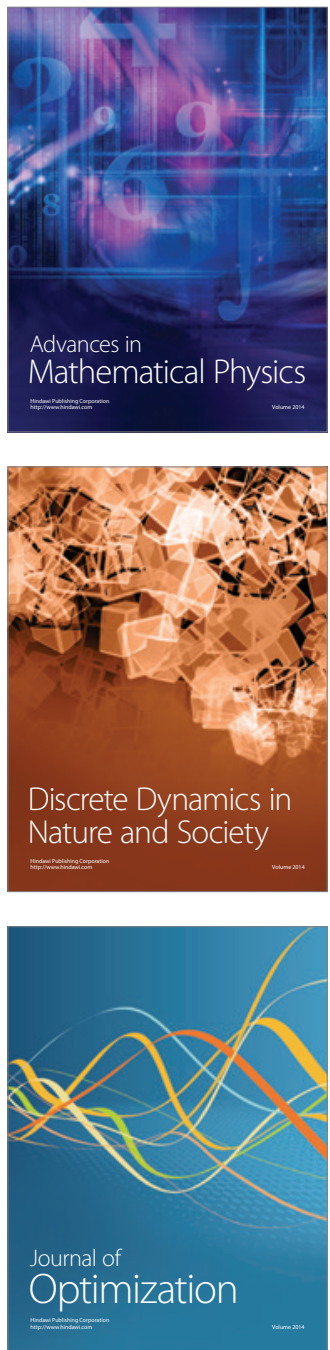